Cite this: Soft Matter, 2014, 10, 4208

\title{
Measuring the configurational temperature of a binary disc packing
}

Received 21st December 2013 Accepted 12th March 2014

DOI: $10.1039 / \mathrm{c} 3 \mathrm{sm} 53176 \mathrm{~g}$

www.rsc.org/softmatter

\begin{abstract}
Song-Chuan Zhao and Matthias Schröter*
Jammed packings of granular materials differ from systems normally described by statistical mechanics in that they are athermal. In recent years a statistical mechanics of static granular media has emerged where the thermodynamic temperature is replaced by a configurational temperature $X$ which describes how the number of mechanically stable configurations depends on the volume. Four different methods have been suggested to measure $X$. Three of them are computed from properties of the Voronoi volume distribution, the fourth takes into account the contact number and the global volume fraction. This paper answers two questions using experimental binary disc packings: first we test if the four methods to measure compactivity provide identical results when applied to the same dataset. We find that only two of the methods agree quantitatively. This implies that at least two of the four methods are wrong. Secondly, we test if $X$ is indeed an intensive variable; this becomes true only for samples larger than roughly 200 particles. This result is shown to be due to recently measured correlations between the particle volumes [Zhao et al., Europhys. Lett., 2012, 97, 34004]
\end{abstract}

\section{Is there a well defined configurational temperature?}

Temperature is the concept that helps us to understand how the exchange of energy stored in the microscopic degrees of freedom follows from the accompanying change of entropy of the involved systems. If we coarse-grain our view to the macroscopic degrees of freedom of particulate systems, such as foams or granular gases, we can still define effective temperatures that describe their dynamics. ${ }^{1,2}$ This approach defines these systems as dissipative; the kinetic energy of the particles is irrecoverably lost to microscopic degrees of freedom.

In the absence of permanent external driving such a system will always evolve towards a complete rest. In the presence of boundary forces or gravity this rest state will be characterized by permanent contacts between the particles which allow for a mechanical equilibrium. Shahinpoor ${ }^{3}$ and Kanatani $^{4}$ were the first to suggest that such systems might still be amenable to a statistical mechanics treatment. Sam Edwards and co-workers ${ }^{5,6}$ have then developed this idea into a full statistical mechanics of static granular matter by using the ensemble of all mechanically stable states as a basis. A necessary requirement for such an approach is the existence of some type of excitation which lets the system explore the phase space of the possible static configurations. This could e.g. be realized by tapping, cyclic shear, or flow pulses of the interstitial liquid. While there are

Max Planck Institute for Dynamics and Self-Organization (MPIDS), 37077 Goettingen, Germany.E-mail: matthias.schroeter@ds.mpg.de promising results, the feasibility of this approach is still under debate. $^{7}$

A second key concept in Edwards' approach is the replacement of the energy phase space by a volume phase space where the volume function $W(\mathbf{q})$ takes the role of the classical Hamiltonian. The configuration q represents the positions and orientations of all grains. One can then define an analog to the partition function $Z(X)$ :

$$
Z(X)=\int \mathrm{e}^{-W(\mathbf{q}) / X} \Theta(\mathbf{q}) \mathrm{d} \mathbf{q}
$$

where $\Theta(\mathbf{q})$ limits the integral to mechanically stable configurations. $X$ is the configurational temperature or compactivity, which is defined as $X=\partial V / \partial S$. The configurational entropy $S$ corresponds to the logarithm of the number of mechanically stable configurations for a given set of boundary conditions.

$S$ is neither known from first principles (except for model systems $\left.^{8,9}\right)$ nor can it be measured directly. Therefore "thermometers" measuring $X$ have to exploit other relationships; four different ways to do so have been suggested. In this paper we will test all four of them using the same dataset of mechanically stable disc packings.

First $X$ can be determined from the steady state volume fluctuations using an analog to the relationship between specific heat and energy fluctuations; ${ }^{10-13}$ we will refer to this compactivity as $X_{\mathrm{VF}}$. A second method is based on the probability ratio of overlapping volume histograms, ${ }^{14,15}$ allowing us to compute $X_{\mathrm{OH}}$. A third way ${ }^{16,17}$ computes $X_{\Gamma}$ from the Gamma function fits to the volume distribution. Finally, it has been suggested recently ${ }^{18}$ that an analysis based on so-called 
quadrons ${ }^{19}$ instead of Voronoi cells leads to an expression for $X_{\mathrm{Q}}$ that involves the average particle volume and the contact number.

This difference in approach immediately raises the question if these four methods provide identical results when applied to the same experiment. There have been two previous experiments addressing this question: McNamara et al. ${ }^{15}$ found that $X_{\mathrm{VF}}$ and $X_{\mathrm{OH}}$ agree for tapped packings of approximately 2000 glass spheres. The same result has been reported by Puckett and Daniels $^{\mathbf{2 0}}$ for compressed disc packings.

A full description of a granular packing has to take the boundary stresses into account. ${ }^{18,21,22}$ The stress dependence of the entropy then gives rise to a tensorial temperature named angoricity. Angoricity has been computed from numerics using the overlapping histogram method ${ }^{23}$ and from experiments using both fluctuations and overlapping histograms. ${ }^{20}$ As the experiments described below are performed in an open cell with gentle driving, the boundary stresses can be assumed to be small and constant; we therefore exclude angoricity from our further analysis.

\section{Correlations in the Voronoi-volume of disc packings}

The three methods to compute $X_{\mathrm{VF}}, X_{\mathrm{OH}}$, and $X_{\Gamma}$ all start from the Voronoi-volume distribution. However, it has been shown that the Voronoi volumes inside a sample are correlated. ${ }^{25} \mathrm{We}$ recently measured the spatial extension of these correlations and demonstrated the existence of additional anti-correlations between the volumes. ${ }^{24}$ These correlations raise the question if $X$ is indeed an intensive parameter i.e. if its value is independent of the number $N$ of particles analyzed. As the Voronoivolumes reported in ref. 24 will be the basis of this study we quickly recap the relevant experimental procedures and results, more details can be found in the original publication. The disc coordinates of all configurations and volume fractions can be downloaded from the Dryad repository. ${ }^{26}$

\subsection{Experimental setup}

The experiment is performed in an air-fluidized bed filled with a binary mixture of Teflon discs with $d_{\mathrm{s}}=6 \mathrm{~mm}$ and $d_{\mathrm{l}}=9 \mathrm{~mm}$ diameter. Datasets of 8000 different mechanically stable configurations are prepared by repeated air pulses. Changing pressure and duration of the air pulses allows us to control the average packing fractions $\phi$ in the ranges of 0.818 to 0.838 . After each flow pulse the discs come to a complete rest and are then imaged with a CCD camera (Fig. 1a). After detecting the particle centers, the Voronoi volume $V$ of each disc is determined (Fig. 1b). We then compute the free volume $V_{\mathrm{f}}=V-V_{\min }$ for each particle with $V_{\min }$ being the volume the grain would occupy in a hexagonal packing of identical discs. This step allows us to superimpose the results for small and large discs in the subsequent analysis.

Our results will also depend on the packing fraction of the loosest possible packing $\phi_{\mathrm{RLP}}=0.811$. This value is averaged
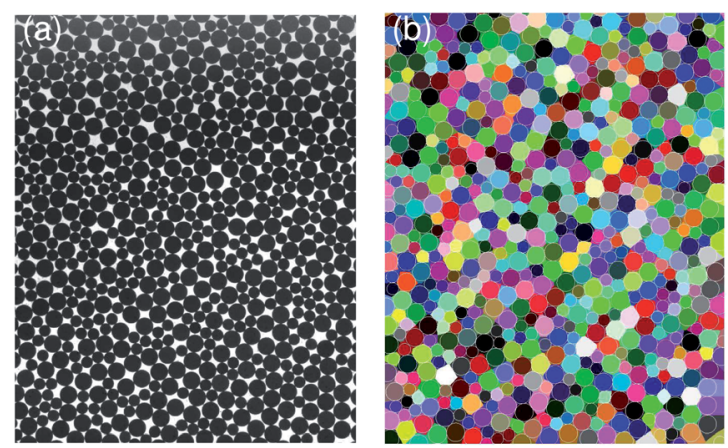

(c)

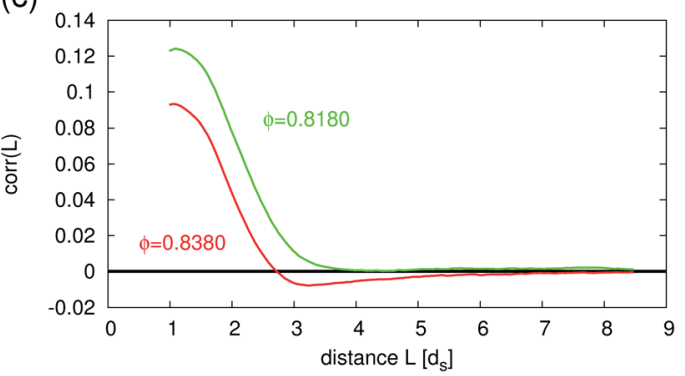

Fig. 1 Correlations in binary disc packings. (a) Experimental image and (b) the corresponding Voronoi tessellation. (c) The correlation between two Voronoi volumes as a function of their distance $L$ (measured in units of small disc diameters $d_{\mathrm{s}}$ ). Dense packings exhibit anti-correlations for $L$ larger than approx. $2.5 d_{\mathrm{s}}$. Reproduced from ref. 24

over 10 packings prepared by slowly settling the discs in a manually decreased air flow.

\subsection{Correlations in binary disc packings}

The correlation between the Voronoi volumes can be measured using:

$$
\operatorname{corr}(L)=\frac{\left\langle\left(V_{\mathrm{f}, i}-\bar{V}_{\mathrm{f}, i}\right)\left(V_{\mathrm{f}, j}-\bar{V}_{\mathrm{f}, j}\right)\right\rangle}{\sigma_{\mathrm{f}}{ }^{2}}
$$

Here $i$ and $j$ are two points in the packing which are separated by a distance $L$. The free volumes at these points are $V_{\mathrm{f}, i}$ and $V_{\mathrm{f}, j}$. Subtracted from them is the average free volume $\bar{V}_{\mathrm{f}}$ of all particles at this point (averaged over all 8000 taps). $\langle\ldots\rangle$ indicates averaging over the 8000 packings and additionally 240 pairs of points $i, j$ within each packing. $\sigma_{\mathrm{f}}^{2}$ is the variance of the free volumes averaged over the two points.

Fig. 1c depicts $\operatorname{corr}(L)$ for two different packing fractions. At low values of $\phi$ only positive correlations between Voronoi cells are found. Above $\phi_{\mathrm{AC}}=0.8277$ anti-correlations appear for $L$ larger than approximately 2.5 small particle diameters. We will show below that these (anti-) correlations control how $X$ becomes intensive.

\section{Compactivity $X_{\mathrm{VF}}$ measured from volume fluctuations}

This methods starts from the assumption of a Boltzmann like probability distribution: 


$$
p(\mathbf{q}, X)=\frac{\mathrm{e}^{-W(\mathbf{q}) / X}}{Z(X)} \Theta(\mathbf{q})
$$

From eqn (3) follows the probability to observe a certain volume $V$ at a given compactivity $X$ :

$$
\begin{aligned}
p(V, X) & =\int \delta(W(\mathbf{q})-V) p(\mathbf{q}, X) \mathrm{d} \mathbf{q} \\
& =\mathscr{D}(V) \frac{\mathrm{e}^{-V / X}}{Z(X)}
\end{aligned}
$$

where the density of states $\mathscr{D}(V)=\int \delta(W(\mathbf{q})-V) \Theta(\mathbf{q})$ dq counts the number of the mechanical stable states available at volume $V$. Using eqn (4) we can determine the average volume $\bar{V}(X)$ as

$$
\bar{V}(X)=\int \mathscr{D}(V) \frac{\mathrm{e}^{-V / X}}{Z(X)} V \mathrm{~d} V
$$

Taking the derivative of eqn (5) with respect to $1 / X$ shows that

$$
\frac{\mathrm{d} \bar{V}}{\mathrm{~d}(1 / X)}=-(V-\bar{V})^{2}=-\sigma_{\bar{V}}^{2}
$$

on the other hand $\mathrm{d} \bar{V} / \mathrm{d}(1 / X)$ can be rewritten as $-X^{2} \mathrm{~d} \bar{V} / \mathrm{d} X$ from which follows:

$$
X^{2} \frac{\mathrm{d} \bar{V}}{\mathrm{~d} X}=\sigma_{\bar{V}}^{2}
$$

Nowak et al. were the first to suggest that integrating eqn (7) provides a way to compute $X:^{\mathbf{1 0}}$

$$
\frac{1}{X_{\mathrm{VF}}}=\int_{\bar{V}}^{\bar{V}_{\mathrm{RLP}}} \frac{\mathrm{d} \bar{V}}{\sigma_{\bar{V}}^{2}}
$$

where the compactivity at the loosest possible packing has been set to infinity: $X\left(\phi_{\mathrm{RLP}}\right)=\infty$. First measurements ${ }^{\mathbf{1 1 , 1 2}}$ of $X_{\mathrm{VF}}$ showed that it indeed measures a material property as it depends on the roughness of the particles. It has to be pointed out that this method has the epistemological status of a calculation rule and not of a test of the Boltzmann assumption made in eqn (3). This is different for the overlapping histogram method described below.

As described in Section 2 we are interested in the evolution of $X$ with the size of the analyzed region, in the following referred to as a cluster. Therefore we continue our study by using the normalized average volume per particle $v=1 / N \sum V / V_{\mathrm{g}}$ where the sum goes over all $N$ particles inside the cluster, $V$ is the Voronoi volume of the individual particles and $V_{\mathrm{g}}$ the volume occupied by the particle itself. As a consequence of this choice also $X$ is dimensionless.

Fig. 2a shows that for our tapped disc packings the variance of the volume fluctuation ${\sigma_{\bar{v}}}^{2}$ increases monotonically with the average volume $\bar{v}$ (bar meaning again the average over all 8000 taps). To compute $X_{\mathrm{VF}}$ we perform a power law fit to ${\sigma_{\bar{v}}}^{2}$ and use the result to integrate eqn (8) numerically. Fig. 2b shows how the resulting $X_{\mathrm{VF}}$ depends on the packing fraction at cluster (a)


Fig. 2 Compactivity $X_{V F}$ measured from volume fluctuations. (a) Average volume variance $\sigma_{\bar{v}}{ }^{2}$ versus average volume $\bar{v}$ measured for clusters of size $N=10$ and 150 . The solid curves are power law fits which are then used to numerically integrate eqn (8). (b) The compactivity $X_{\mathrm{VF}}$ computed from eqn (8) for three different cluster sizes. (c) The evolution of $K_{\mathrm{VF}}$ with cluster sizes at different values of $\phi$. The radius $R$ of the analyzed cluster is proportional to $\sqrt{N / \phi}$.

sizes $N$ of 1,30 , and 150 discs. It is obvious that it is not an intensive variable for this range of $N$.

\subsection{Correlations make $X_{\mathrm{VF}}$ non-intensive in small systems}

For a more detailed analysis we have plotted in Fig. $2 \mathrm{c}$ the dependence of $X_{\mathrm{VF}}$ on the cluster radius $R$ measured from small disc diameters $d_{\mathrm{s}}$. Three features of $X_{\mathrm{VF}}$ become apparent:

(1) $X_{\mathrm{VF}}$ is growing monotonously for $R<3 d_{\mathrm{s}}$ for all values of $\phi$,

(2) for low to intermediate values of $\phi, X_{\mathrm{VF}}$ then reaches a plateau, and

(3) for the highest packing fraction $X_{\mathrm{VF}}$ first decreases slightly before entering a plateau.

All three points can be understood by considering the influence of the volume correlations shown in Fig. 1. Eqn (8) computes $X_{\mathrm{VF}}$ from the average variance ${\sigma_{\bar{v}}}^{2}$ inside the cluster. This variance can be decomposed in the following way:

$$
{\sigma_{\bar{v}}}^{2}=\frac{1}{N} \sum_{k}^{N} \sum_{m}^{N}\left\langle\delta v_{k} \delta v_{m}\right\rangle=\frac{1}{N} \sum_{k}^{N}\left(\sigma_{k}^{2}+\sum_{m \neq k}\left\langle\delta v_{k} \delta v_{m}\right\rangle\right)
$$

Here $\sigma_{k}^{2}=\left\langle\delta v_{k}^{2}\right\rangle$ is the fluctuation of a single Voronoi cell and $\sum_{m \neq k}\left\langle\delta v_{k} \delta v_{m}\right\rangle:=I_{k}$ is the volume correlation between disc $k$ and all other discs inside the cluster. If $k$ is in the center of the 
cluster, then $I_{k}$ is proportional to the area enclosed by $\operatorname{corr}(L)$, the zero line, and $L=R$ in Fig. 1c.

This consideration allows us to explain feature 1: while $N$ goes from 2 to approximately 25 all $I_{k}$ values are positive and growing. Consequentially, the variance of the cluster ${\sigma_{\bar{v}}}^{2}$ is larger than the sum of the variance of the individual Voronoi cells $1 / N \sum \sigma_{k}^{2}$ and $X_{\mathrm{VF}}$ grows monotonously with $N$.

The second feature stems from the fact that once disk $k$ is more than four to five $d_{\mathrm{s}}$ away from the boundary of the cluster, $I_{k}$ becomes independent of the cluster size. As the relative importance of "boundary discs" decreases with the cluster size, $\sigma_{\bar{v}}^{2}$ becomes constant and $X_{\mathrm{VF}}$ becomes independent of $N$.

Finally, the slight decrease in $X_{\mathrm{VF}}$ at high packing fractions and $N$ values between approximately 30 and 150 can be attributed to the anti-correlations that appear for $\phi$ larger than 0.8277 ; these will decrease $I_{k}$ slightly again before it reaches its plateau value.

\section{Compactivity $X_{\mathrm{OH}}$ measured from overlapping histograms}

This way to compute compactivity has been first described by Dean and Lefèvre. ${ }^{14}$ It uses pairs of experiments with slightly different values of $\phi$, respectively $X_{\mathrm{OH}}$, and computes then the ratio of the probabilities to observe the same local volume $V$. If the assumption of a Boltzmann-like probability distribution, as expressed in eqn (4), holds, this ratio should be exponential in $V$ :

$$
\frac{p\left(V, X_{1}\right)}{p\left(V, X_{2}\right)}=\frac{\frac{\mathscr{D}(V) \mathrm{e}^{-V / X_{1}}}{Z\left(X_{1}\right)}}{\frac{\mathscr{D}(V) \mathrm{e}^{-V / X_{2}}}{Z\left(X_{2}\right)}}=\frac{Z\left(X_{2}\right)}{Z\left(X_{1}\right)} \mathrm{e}^{-\left(\frac{1}{X_{1}}-\frac{1}{X_{2}}\right) V}
$$

By taking the logarithm on both sides we obtain:

$$
Q:=\ln \frac{p_{X_{1}}(V)}{p_{X_{2}}(V)}=\left(\frac{1}{X_{2}}-\frac{1}{X_{1}}\right) V+\ln \frac{Z_{X_{2}}}{Z_{X_{1}}}
$$

Therefore the difference between two compactivities can be computed from a line fit of $Q$ versus $V$ as it was first demonstrated in ref. 15 .

Fig. 3a shows the distribution of average volumes for two experiments with a packing fraction difference of 0.0017 . Fig. 3b demonstrates that the ratio $Q$ is indeed a linear function of $V$, as predicted by eqn (11). By sweeping the experimentally accessible range of packing fractions, $X_{\mathrm{OH}}$ can be determined from the accumulated compactivity differences up to an additive constant $X_{0}$. We determine $X_{0}$ by setting $X_{\mathrm{OH}}$ for the loosest experimental packing to the value of $X_{\mathrm{VF}}$ at this volume fraction.

The resulting $X_{\mathrm{OH}}$ is shown in Fig. 4. The good quantitative agreement of $X_{\mathrm{OH}}$ and $X_{\mathrm{VF}}$ is not too surprising given that (a) both methods are derived from the same probability distribution (eqn (4)) and (b) our determination of $X_{0}$. However, the $X_{\mathrm{OH}}$ method provides an additional test of the assumptions leading to eqn (4) as we can compare the quality of a linear fit to $Q(V)$ (a)

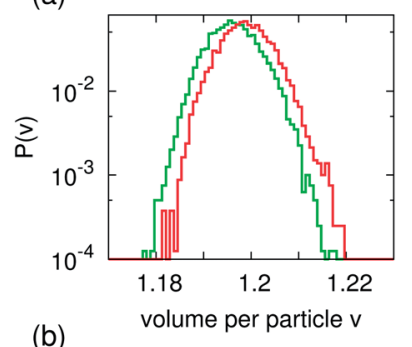

(b)

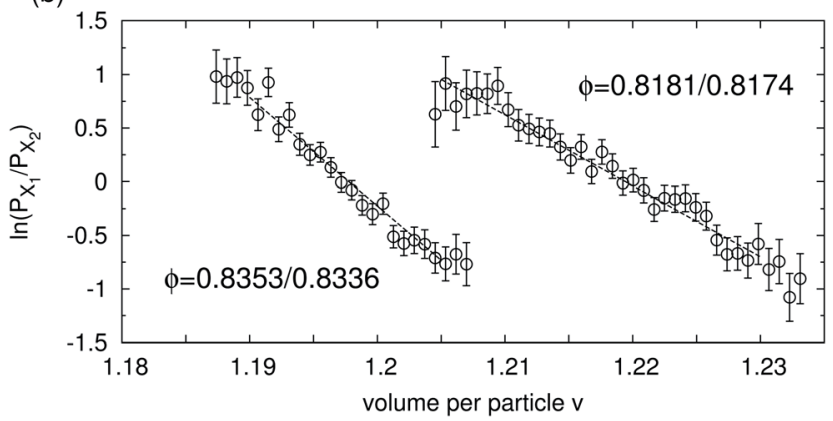

(c)

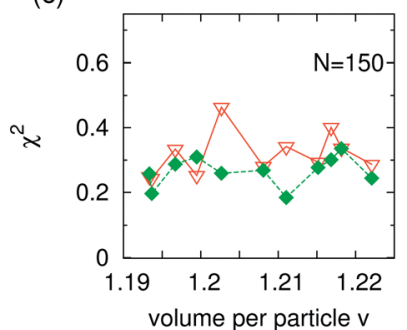

Fig. 3 Compactivity measured with the overlapping histogram method. (a) The probability to observe an average volume per particle in clusters with 150 disks. The average packing fraction corresponds to 0.8336 for the red curve, and 0.8353 for the green curve. (b) The logarithm of the probabilities to observe a given volume at two different compactivities respectively packing fractions is a linear function of the volume, which is in accordance with eqn (11). The cluster size is 150 discs, and the dashed lines are linear fits. (c) The $\chi^{2}$ values provide a goodness of fit test for both linear (green diamonds) and parabolic (red triangle) fits to the probability ratios shown in panel b. The average $\chi^{2}$ is $13 \%$ smaller for the linear fit, indicating that a Boltzmann-like distribution is a better assumption than a Gaussian.



Fig. 4 Comparison of $X_{\mathrm{OH}}, X_{\mathrm{VF}}$, and $X_{\Gamma}$ computed for single discs and clusters of 150 particles. In all three cases the compactivity of an individual particle is smaller than that of a larger cluster. $X_{\mathrm{OH}}$ and $X_{\mathrm{VF}}$ agree quantitatively, $X_{\Gamma}$ is about an order of magnitude smaller.

with fit functions derived from alternative probability distribution functions. ${ }^{15}$ A generic candidate would be a Gaussian distribution which results in a parabolic fit. Fig. 3c demonstrates however that a linear fit is superior, adding credibility to a Boltzmann-like approach. Also note that canceling the density of states in eqn (10) implicitly assumes a weak form of 
ergodicity; if the system explores its phase space differently at the two values of $X$ we cannot eliminate $\mathscr{D}(V)$.

\section{Compactivity $X_{\Gamma}$ measured from a Gamma distribution fit}

This third method to compute compactivity has been suggested by Aste and Di Matteo; ${ }^{17,27}$ it differs from the previous approaches that it explicitly determines the density of states $\mathscr{D}(V)$. Based on the observation ${ }^{\mathbf{1 6}}$ that experimental Voronoi volume distributions can be well fit by $\Gamma$ distributions, they propose to replace eqn (4) with a rescaled $k$-Gamma distribution

$$
p\left(V_{\mathrm{f}}\right)=\left(\frac{k}{\bar{V}_{\mathrm{f}}}\right)^{k} \frac{V_{\mathrm{f}}^{(k-1)}}{\Gamma(k)} \exp \left(-k \frac{V_{\mathrm{f}}}{\bar{V}_{\mathrm{f}}}\right)
$$

where $\bar{V}_{\mathrm{f}}$ is the mean free volume (as defined in Section 2), and $k$ is the shape factor. They then identify

$$
X_{\Gamma}=\frac{\bar{V}_{\mathrm{f}}}{k}
$$

or by making use of the fact that variance ${\sigma_{\bar{V}_{\mathrm{f}}}}^{2}$ of the $\Gamma$ function is given by: $\sigma_{\bar{V}_{\mathrm{f}}}^{2}=\bar{V}_{\mathrm{f}}^{2} / k$ they derive

$$
X_{\Gamma}=\frac{\sigma_{\bar{V}_{\mathrm{f}}}^{2}}{\bar{V}_{\mathrm{f}}}
$$

By comparing eqn (4) and (12) we can also identify the density of states:

$$
\frac{\mathscr{D}(V)}{Z(X)}=X_{\Gamma}^{-k} \frac{V_{\mathrm{f}}^{(k-1)}}{\Gamma(k)}
$$

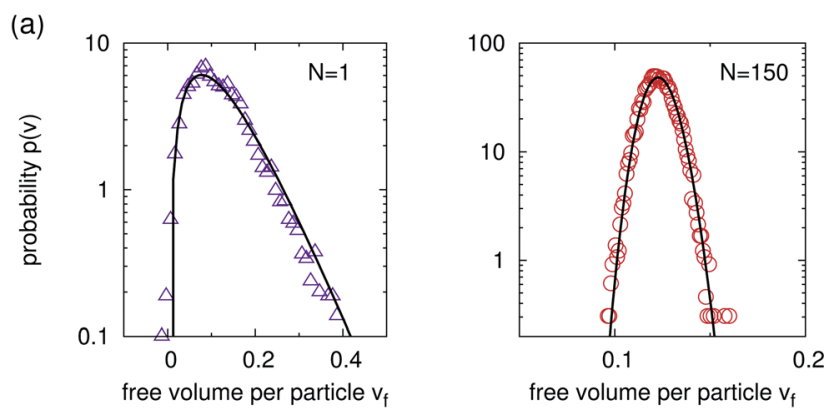

(b)

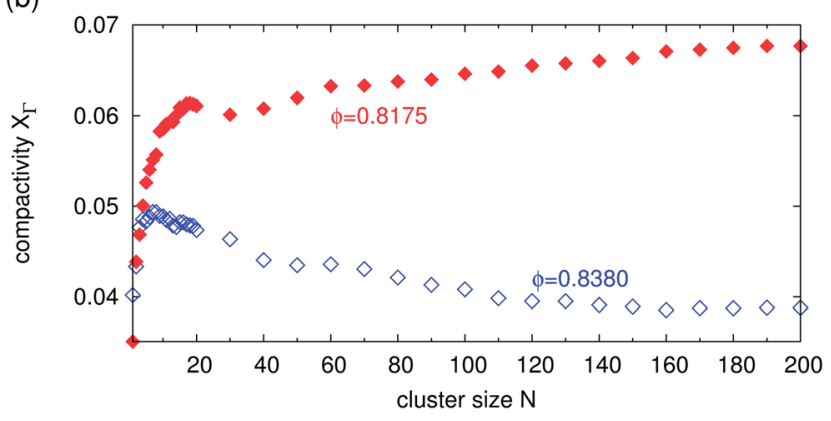

Fig. 5 Applying the $\Gamma$ distribution method. (a) $\Gamma$ distribution fits to the volume distributions for a single disc and 150 particle clusters $(\phi=$ 0.8175). (b) Evolution of $X_{\Gamma}$ with the cluster size.
Fig. 5a demonstrates that our volume distribution is well fit by a $\Gamma$ distribution. ${ }^{28}$ We then determine $X_{\Gamma}$ without any additive constant using eqn (14). Fig. 4 shows $X_{\Gamma}$ in comparison to $X_{\mathrm{VF}}$ and $X_{\mathrm{OH}}$. While all three compactivities decrease monotonously with $\phi$, the absolute values and the slope of $X_{\Gamma}$ are quite different from $X_{\mathrm{VF}}$ and $X_{\mathrm{OH}}$.

Fig. 5b shows the evolution of $X_{T}$ with the cluster size. A comparison with $X_{\mathrm{VF}}$, displayed in Fig. 2c, shows a qualitative similar influence of the volume correlations: while $X_{\Gamma}$ increases towards a plateau for small values of $\phi$, it goes through a maximum before reaching a smaller plateau value for the densest packings.

\section{Compactivity $X_{\mathrm{Q}}$ measured from quadron tessellation}

In a recent paper Blumenfeld et al. ${ }^{18}$ presented an analysis of the statistical mechanics of two-dimensional packings based on quadrons $^{19}$ as the building blocks of the tessellation. An advantage of this choice is that the quadrons take by design into account the structural degrees of freedom of the individual particles. A drawback is that quadrons are not necessarily volume conserving in the presence of non-convex voids formed by "rattlers", i.e. particles lying at the bottom of larger voids. ${ }^{29,30}$ Blumenfeld and co-workers then derive the partition functions
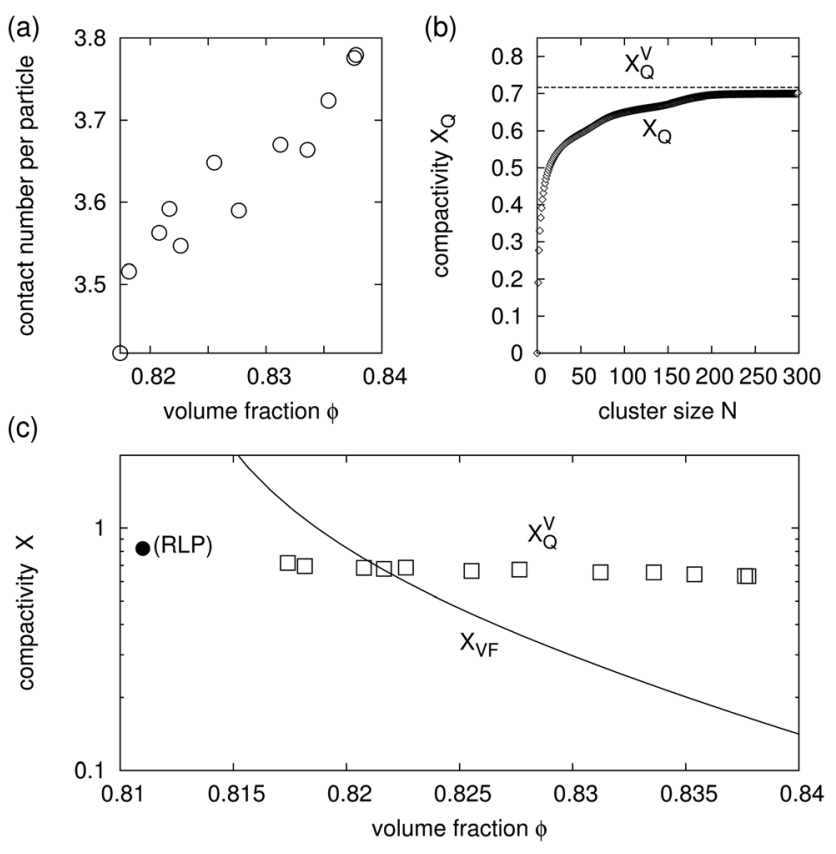

Fig. 6 Compactivity measured with the quadron tessellation method. (a) In all our experiments the contact number per particle is above the isostatic value of 3. (b) A comparison of $X_{Q}$ computed from the full partition function (eqn (16)) and $X_{Q}^{V}$ derived from the volume ensemble (eqn (17)) only. Both are computed for $\phi=0.8175$. The difference vanishes in the large system limit. (c) A comparison of $X_{\mathrm{VF}}$ (measured for $N=150$ ) and $X_{Q}^{V}$. The filled circle is computed for our random loose packing value which is presumably the only isostatic point in our dataset. The open squares assume that eqn (16) is also valid for hyperstatic packings. 
of the volume ensemble $Z_{\mathrm{V}}$, the force ensemble $Z_{\mathrm{F}}$, and the total partition function $Z$, showing in this process that $Z \neq Z_{\mathrm{V}} Z_{\mathrm{F}}$. Finally, they obtain from $Z$ an expression for $X_{\mathrm{Q}}$ of an isostatic packing:

$$
X_{\mathrm{Q}}=\frac{2}{\bar{z} N+2 M}\langle V\rangle
$$

$\langle V\rangle$ is the average volume of a cluster, $\bar{z}$ the average number of contacts a particle has, and $M$ is the number of boundary forces.

If $X_{\mathrm{Q}}^{V}$ is derived from $Z_{\mathrm{V}}$ instead of $Z$ one obtains:

$$
X_{\mathrm{Q}}^{V}=\frac{2}{\bar{z} N}\langle V\rangle
$$

In comparing our results with this approach we have to acknowledge two differences. First, our experimental packings are clearly subject to volume forces due to gravity. And secondly they are, as shown in Fig. 6a, hyperstatic, i.e. their contact number is larger than what is required to fix all their mechanical degrees of freedom. ${ }^{31}$

Generally, packings of frictional particles only become isostatic at random loose packing and zero pressure. ${ }^{32,33}$ Therefore the only direct comparison possible is at our RLP value $\phi=0.811$, the corresponding $X_{\mathrm{Q}}$ is shown as a black circle in Fig. 6c. If we assume that we can replace $\bar{z}$ in eqn (16) with $\bar{z}(\phi)$ and use the contact numbers displayed in Fig. 6a, we can also compute $X_{\mathrm{Q}}$ for a larger range of $\phi$. These results are indicated as open squares in Fig. 6c.

As $X_{\mathrm{Q}}$ is only computed from the average volume of a cluster, it is insensitive to the correlations described in Section 2. On the other hand there exists a finite size effect if $X_{\mathrm{Q}}^{V}$ is computed from the volume ensemble, ignoring the boundary forces. Fig. $6 \mathrm{~b}$ shows how the difference between the compactivities computed from the full and the volume ensemble vanishes with increasing cluster size and consequentially decreasing contribution of $M$.

\section{Which is the correct way to measure compactivity?}

Fig. 4 and $6 \mathrm{c}$ show clearly that the four different methods to compute compactivity do not agree quantitatively. While $X_{\mathrm{OH}}$ and $X_{\mathrm{VF}}$ are identical within experimental scatter, $X_{\Gamma}$ is more than one order of magnitude smaller than $X_{\mathrm{OH}}$ and $X_{\mathrm{VF}}$ and at the same time decreasing less steeply with volume fraction. ${ }^{34}$ The values of $X_{\mathrm{Q}}$ are closer to $X_{\mathrm{OH}}$ and $X_{\mathrm{VF}}$ again, but the evolution with $\phi$ is similar to $X_{\Gamma}$.

Our data can be used to self-consistently compute all four different versions of compactivity, consequentially they are unsuitable as a basis to judge the correctness of the different approaches. A potential experimental or numerical test would need an independent determination of the compactivity of a model system from the knowledge of its entropy as a function of volume. Alternatively, if the granular statistical mechanics could be advanced to make predictions of granular behavior (e.g. segregation ${ }^{35}$ ) based on specific values of $X$, these could be tested versus the four different "thermometers".

However, we can elucidate the difference between the four methods by computing the density of states and comparing it to what is known about RLP, the loosest possible, mechanically stable packing.

\subsection{Computing the density of states}

A probability distribution of the type of eqn (3) is a generic feature for every statistic theory that maximizes the amount of entropy represented by $p$, given certain constraints. ${ }^{36}$ In this spirit the density of states $\mathscr{D}(V)$ in eqn (4) can be interpreted as encapsulating the physics of the specific system under consideration, while the exponential term represents our lack of knowledge about the microscopic state. From this perspective the main difference between the four methods is the way they determine $\mathscr{D}(V)$ : while $X_{\mathrm{VF}}$ and $X_{\mathrm{OH}}$ treat it as an experimental input parameter, $X_{T}$ and $X_{\mathrm{Q}}$ do provide predictions for its dependence on $V$. In this subsection we will however not discuss $X_{\mathrm{Q}}$ as the theory has not been expanded yet to hyperstatic packings.

$\mathscr{D}(V)$ can be computed following McNamara et al. ${ }^{15}$ Starting by rewriting eqn (4) to

$$
\mathscr{D}(V)=p(V, X) \mathrm{e}^{V / X} Z(X),
$$

we remove the dependence on the unknown partition function by taking the ratio with $D$ at a given volume $V_{0}$ :

$$
\frac{\mathscr{D}(V)}{\mathscr{D}\left(V_{0}\right)}=\frac{p(V, X)}{p\left(V_{0}, X\right)} \mathrm{e}^{1 / X\left(V-V_{0}\right)}
$$

The results are presented in Fig. 7 where we have chosen $V_{0}=1.2$. Panel (a) shows that the density of state ratios computed from all eleven values of $X_{\mathrm{OH}}$ overlap, as it can be expected if the different preparation protocols sample the phase space with the same probabilities. Fig. 7b shows that this agreement of the density of states is not obtained if the ratio is computed from $X_{\Gamma}$.

Fig. 7 provides also some insight into how the configurational entropy $S$ depends on the volume:

$$
S=k_{\mathrm{E}} \ln (\mathscr{D}(V) \delta V)=k_{\mathrm{E}} \ln \mathscr{D}(V)+k_{\mathrm{E}} \ln \delta V
$$

where $k_{\mathrm{E}}$ is the equivalent to the Boltzmann constant. As our system is large enough and not constrained by boundaries (for a counter example see ref. 37), we obtain a smooth function $\mathscr{D}(V)$ and can therefore choose the integration interval $\delta V$ small enough so that its contribution to $S$ vanishes.

\subsection{Random loose packing}

Random Loose Packing (RLP) is first of all defined phenomenologically as the loosest possible packing which is still mechanically stable, i.e. has a finite yield stress. Mechanical stability requires the packing to be at least isostatic: the average number of contacts of a particle needs to be large enough to provide sufficient constraints to fix all its degrees of freedom. ${ }^{32,33,38}$ Experimentally it has been found that $\phi_{\mathrm{RLP}}$ of 

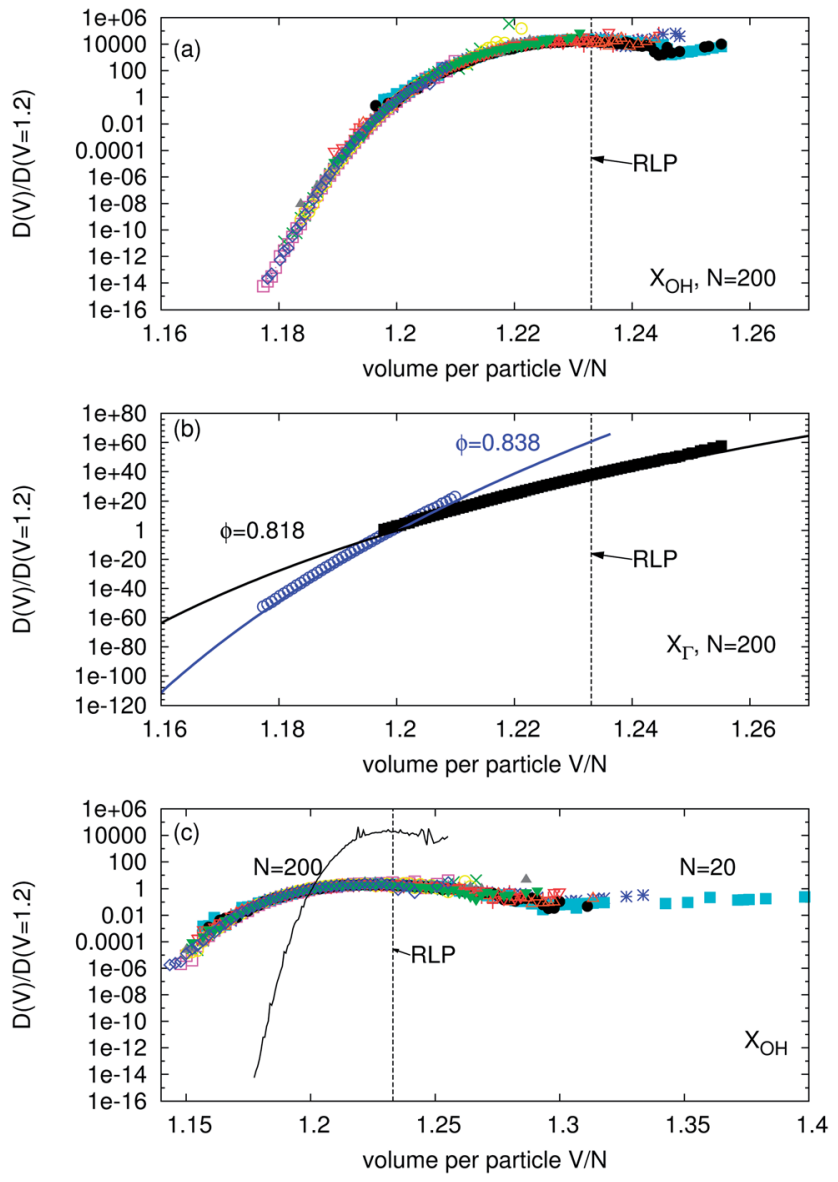

Fig. 7 The density of states ratio. Panel (a) is based on the eleven measurements of $X_{\mathrm{OH}}$ for the cluster size $N=200$. The dashed line indicates the global random loose packing volume. Panel (b) is computed from $X_{T}$ of the densest and loosest packings. Points represent the measured distributions, solid lines are analytical results based on the Gamma functions (eqn (15)). In contrast to panel (a) the two curves do not overlap. (c) The density of states depends on the cluster size. The solid, black curve corresponds to the average of all data points with $N=200$ in panel (a), the individual data points are computed from $X_{\mathrm{OH}}$ measured at $N=20$.

spheres is approximately 0.55 (ref. 39-42) with the precise value depending on the friction coefficient $\mu$ (ref. 40-42) and the confining pressure. ${ }^{40}$ For binary disc packings $\phi_{\mathrm{RLP}}$ also depends on their diameter ratio; for the particles in this paper we measured $\phi_{\mathrm{RLP}}=0.811$.

While RLP seems to imply that there exist no packings with lower $\phi$, exactly such states have been identified in MD simulations with $N=20$ discs; the new lower boundary where such states vanish has been named Random Very Loose Packing (RVLP). ${ }^{43}$ An explanation why these states between RLP and RVLP are usually not observed in experiments is given by their small basin of attraction in phase space.

In general, the statistical mechanics approaches to granular media $^{5-7}$ assume the configurational temperature $X \geq 0$. Then it follows from

$$
\partial S=1 / X \partial V
$$

that as the configurational entropy increases the packings become looser. At RLP $S$ reaches a maximum and then decays to zero for looser configurations which by definition are not mechanically stable packings. Further on, it is often assumed that the maximum of $S$ is analytic which implies that $X$ becomes infinity at RLP. ${ }^{5,6,43}$

The density of states computed from $X_{\mathrm{VF}}$, respectively $X_{\mathrm{OH}}$, agrees with these predictions; Fig. 7 shows that it reaches a global maximum at RLP. This is however only a self-consistency test as the value of $\phi_{\mathrm{RLP}}$ explicitly entered the computation of these two compactivities. More probative is the fact that the volume fluctuations used to compute $X_{\mathrm{VF}}$ have been shown to depend on $\mu .^{11}$ Consequentially, also a configurational entropy computed from $X_{\mathrm{VF}}$ will depend on $\mu$, which is a necessary condition as the number of mechanically stable configurations also depends on $\mu$.

Fig. 7c shows that the density of states also depends on the size of the system. Systems with 20 discs posses considerably looser states than those with 200 discs. This effect points to an explanation of the configurations between RLP and RVLP as a consequence of the finite size of a system, they will vanish in the thermodynamic limit.

The density of states computed from $X_{\Gamma}$ (Fig. 7b) seems not to display a maximum at $\phi_{\mathrm{RLP}}$. However, RLP might become the most likely state if in the limit $N \rightarrow \infty$ the states between RLP and RVLP vanish. Either way, $X_{\Gamma}\left(\phi_{\mathrm{RLP}}\right)$ will be finite and display a jump to approximately half its value for the "fluid" configurations below $\phi_{\mathrm{RLP}}{ }^{27}$ which have Voronoi volume distributions that are also well described by Gamma functions. ${ }^{44}$ While this behavior might be not the canonical expectation, especially as $X_{T}$ is undefined for fluid, non-stable configurations, it does not contradict experimental results. This is different for the influence of friction: it has been shown that the Gamma distribution fits are independent of $\mu,{ }^{\mathbf{1 6 , 1 7}}$ consequentially neither $\phi_{\mathrm{RLP}}$ nor $X_{T}$ and the derived $S$ will reflect the different number of mechanical stable states resulting from changes in friction.

Finally, it is difficult to comment on the relationship between $X_{\mathrm{Q}}$ and RLP as the theory is presently only worked out at exactly RLP. $X_{\mathrm{Q}}\left(\phi_{\mathrm{RLP}}\right)$ is of finite value and will depend only very weakly on $\mu$ via $V$. If we allow similar to $X_{T}$ for its existence also for $\phi<\phi_{\mathrm{RLP}}, X_{\mathrm{Q}}$ will be depending on the preparation protocol because the contact number is protocol dependent below RLP. ${ }^{45}$

\subsection{Open questions}

Because granular matter is athermal, the question of ergodicity has to be answered separately for each experimental or numerical protocol used to explore the phase space of mechanically stable configurations. An indication of their differences is e.g. the different $\phi$ dependence of the volume fluctuations when the sample is either mechanically tapped ${ }^{\mathbf{1 2}}$ or excited by flow pulses. ${ }^{\mathbf{1 1}}$ Nonergodicity has recently also been shown for numerical tapping of frictionless hard spheres, ${ }^{\mathbf{4 6}}$ however there the analysis was not restricted to mechanically stable states. In contrast, in numerical simulations of frictional 
discs fluidized with flow pulses an equivalence between time and ensemble averages has been found. ${ }^{47}$

The biggest challenges to the concept of a configurational granular temperature are however recent experiments by Puckett and Daniels ${ }^{\mathbf{2 0}}$ where they studied the volume and force fluctuations of two samples of discs which where in mechanical contact. They found that the angoricity of the two samples equilibrated, but the compactivity (computed as $X_{\mathrm{OH}}$ and $X_{\mathrm{VF}}$ ) did not. However, their preparation protocol was biaxial compression which did not allow for strong particle rearrangements. Therefore, we speculate, it might have been prohibiting volume exchange between the two subsystems by quenching too fast. Clearly more work is needed to answer the question if compactivity is a variable predictive of granular behavior or just a number following from an algorithm.

\section{Conclusions}

Using the same experimental dataset, we have computed the configurational granular temperature $X$ with the four different methods that have been previously suggested. The two methods that treat the density of states as an experimental input agree quantitatively with each other. The $X$ values computed from the other two methods, which specify the density of states from independent theoretical considerations, are both different from each other and from the first two methods. Our results do not provide a direct way to judge the correctness of the individual approaches. But the derived density of states shows that only the two agreeing methods provide a satisfying explanation how random loose packing depends on friction.

Our measurements also demonstrate that $X$ becomes only an intensive variable when computed for clusters of size $N$ larger 150 particles. This effect is due to the volume correlations of neighboring particles. Consequentially, individual Voronoi cells are not suitable 'quasi-particles' to define a configurational temperature in granular packings. This will complicate the application of a statistical mechanics approach to small granular systems and in the presence of local gradients.

\section{Acknowledgements}

We acknowledge helpful discussions with Karen Daniels and Klaus Kassner.

\section{References}

1 I. K. Ono, C. S. OHern, D. J. Durian, S. A. Langer, A. J. Liu and S. R. Nagel, Phys. Rev. Lett., 2002, 89, 095703.

2 N. V. Brilliantov and T. Pöschel, Kinetic Theory of Granular Gases, Oxford University Press, 2004.

3 M. Shahinpoor, Powder Technol., 1980, 25, 163-176.

4 K.-I. Kanatani, Powder Technol., 1981, 30, 217-223.

5 S. Edwards and R. Oakeshott, Phys. A, 1989, 157, 1080-1090. 6 A. Mehta and S. F. Edwards, Phys. A, 1989, 157, 1091-1100.

7 M. Pica Ciamarra, P. Richard, M. Schröter and B. P. Tighe, Soft Matter, 2012, 8, 9731.

8 R. Monasson and O. Pouliquen, Phys. A, 1997, 236, 395-410.
9 R. K. Bowles and S. S. Ashwin, Phys. Rev. E: Stat., Nonlinear, Soft Matter Phys., 2011, 83, 031302.

10 E. R. Nowak, J. B. Knight, E. Ben-Naim, H. M. Jaeger and S. R. Nagel, Phys. Rev. E: Stat., Nonlinear, Soft Matter Phys., 1998, 57, 1971-1982.

11 M. Schröter, D. I. Goldman and H. L. Swinney, Phys. Rev. E: Stat., Nonlinear, Soft Matter Phys., 2005, 71, 030301.

12 P. Ribière, P. Richard, P. Philippe, D. Bideau and R. Delannay, Eur. Phys. J. E: Soft Matter Biol. Phys., 2007, 22, 249-253.

13 C. Briscoe, C. Song, P. Wang and H. A. Makse, Phys. Rev. Lett., 2008, 101, 188001-188004.

14 D. S. Dean and A. Lefèvre, Phys. Rev. Lett., 2003, 90, 198301. 15 S. McNamara, P. Richard, S. K. de Richter, G. Le Car and R. Delannay, Phys. Rev. E: Stat., Nonlinear, Soft Matter Phys., 2009, 80, 031301.

16 T. Aste, T. D. Matteo, M. Saadatfar, T. J. Senden, M. Schröter and H. L. Swinney, EPL, 2007, 79, 24003.

17 T. Aste and T. Di Matteo, Phys. Rev. E: Stat., Nonlinear, Soft Matter Phys., 2008, 77, 021309.

18 R. Blumenfeld, J. F. Jordan and S. F. Edwards, Phys. Rev. Lett., 2012, 109, 238001.

19 R. Blumenfeld and S. F. Edwards, Phys. Rev. Lett., 2003, 90, 114303.

20 J. G. Puckett and K. E. Daniels, Phys. Rev. Lett., 2013, 110, 058001.

21 K. Wang, C. Song, P. Wang and H. A. Makse, Europhys. Lett., 2010, 91, 68001.

22 L. A. Pugnaloni, J. Damas, I. Zuriguel and D. Maza, Pap. Phys., 2011, 3, 030004.

23 S. Henkes, C. S. O'Hern and B. Chakraborty, Phys. Rev. Lett., 2007, 99, 038002.

24 S. Zhao, S. Sidle, H. L. Swinney and M. Schröter, EPL, 2012, 97, 34004 .

25 F. Lechenault, F. d. Cruz, O. Dauchot and E. Bertin, J. Stat. Mech.: Theory Exp., 2006, 2006, P07009.

26 Data available from the Dryad Digital Repository: http:// doi.org/10.5061/dryad.t6m37.

27 T. Aste and T. Di Matteo, Eur. Phys. J. B, 2008, 64, 511-517.

28 The theory leading to $X_{T}$ has been developed for threedimensional monodisperse packings. Our approach of using binary two-dimensional data is justified only empirically by the quality of the Gamma distribution fit (i.e. eqn (12)) to our free volume distribution.

29 M. Pica Ciamarra, Phys. Rev. Lett., 2007, 99, 089401.

30 R. Blumenfeld and S. F. Edwards, Phys. Rev. Lett., 2007, 99, 089402.

31 Two particles are considered to be in contact when the distance between their centers is smaller than 1.01 times the sum of their radii. The prefactor is based on the uncertainty of our image processing, it has been measured from the pair correlation function.

32 K. Shundyak, M. van Hecke and W. van Saarloos, Phys. Rev. E., 2007, 75, 010301.

33 S. Henkes, M. van Hecke and W. van Saarloos, EPL, 2010, 90, 14003. 
34 This difference can not be attributed to the use of the free volume $V_{\mathrm{f}}$ for the computation of $X_{T}$ as $V_{\mathrm{f}}$ differs from $v$ only by a constant $2 \sqrt{3} / \pi$. Therefore neither the shape of the probability distributions used to compute $X_{\mathrm{OH}}$ nor the size of the volume fluctuations, on which $X_{\mathrm{VF}}$ is based on, would change if they were also computed from $V_{\mathrm{f}}$.

35 M. Schröter and K. E. Daniels, arxiv.org/abs/1206.4101.

36 E. T. Jaynes, Phys. Rev., 1957, 106, 620-630.

37 G.-J. Gao, J. Bławzdziewicz and C. S. O’Hern, Phys. Rev. E: Stat., Nonlinear, Soft Matter Phys., 2006, 74, 061304.

38 C. Song, P. Wang and H. A. Makse, Nature, 2008, 453, 629632.

39 G. Y. Onoda and E. G. Liniger, Phys. Rev. Lett., 1990, 64, 2727-2730.
40 M. Jerkins, M. Schröter, H. L. Swinney, T. J. Senden, M. Saadatfar and T. Aste, Phys. Rev. Lett., 2008, 101, 018301. 41 G. R. Farrell, K. M. Martini and N. Menon, Soft Matter, 2010, 6, 2925-2930.

42 L. E. Silbert, Soft Matter, 2010, 6, 2918-2924.

43 M. Pica Ciamarra and A. Coniglio, Phys. Rev. Lett., 2008, 101, 128001.

44 V. Senthil Kumar and V. Kumaran, J. Chem. Phys., 2005, 123, 114501.

45 C. Heussinger and J.-L. Barrat, Phys. Rev. Lett., 2009, 102, 218303.

46 F. Paillusson and D. Frenkel, Phys. Rev. Lett., 2012, 109, 208001.

47 M. Pica Ciamarra, A. Coniglio and M. Nicodemi, Phys. Rev. Lett., 2006, 97, 158001. 